\title{
Numerical Study on Heat Transfer Characteristics of Heated/Cooled Rods Having a Composite Board in between: Effect of Thermal Vias
}

\author{
Yasushi Koito, Toshio Tomimura \\ Division of Energy Science, Faculty of Advanced Science and Technology, Kumamoto University, Kumamoto, Japan \\ Email: koito@gpo.kumamoto-u.ac.jp
}

How to cite this paper: Koito, Y. and Tomimura, T. (2017) Numerical Study on Heat Transfer Characteristics of Heated/Cooled Rods Having a Composite Board in between: Effect of Thermal Vias. Journal of Electronics Cooling and Thermal Control, 7, 91-102. https://doi.org/10.4236/jectc.2017.74008

Received: September 5, 2017

Accepted: November 12, 2017

Published: November 15, 2017

Copyright (๑) 2017 by authors and Scientific Research Publishing Inc. This work is licensed under the Creative Commons Attribution International License (CC BY 4.0).

http://creativecommons.org/licenses/by/4.0/ (c) (i) Open Access

\begin{abstract}
By placing a sample between a heated and a cooled rod, a thermal conductivity of the sample can be evaluated easily with the assumption of a one-dimensional heat flow. However, a three-dimensional constriction/spreading heat flow may occur inside the rods when the sample is a composite having different thermal conductivities. In order to investigate the thermal resistance due to the constriction/spreading heat flow, the three-dimensional numerical analyses were conducted on the heat transfer characteristics of the rods. In the present analyses, a polymer-based composite board having thermal vias was sandwiched between the rods. From the numerical results, it was confirmed that the constriction/spreading resistance of the rods was strongly affected by the thermal conductivity of the rods as well as the number and size of the thermal vias. A simple equation was also proposed to evaluate the constriction/spreading resistance of the rods. Fairly good agreements were obtained between the numerical results and the calculated ones by the simple equation. Moreover, the discussion was also made on an effective thermal conductivity of the composite board evaluated with the heated and the cooled rod.
\end{abstract}

\section{Keywords}

Heat Transfer Enhancement, Thermal Via, Constriction/Spreading

Resistance, Steady-State Method, Effective Thermal Conductivity

\section{Introduction}

A simple way to evaluate a thermal conductivity of a sample is a steady-state 
method using two reference rods of known thermal conductivity [1] [2] [3]. In this method, the sample is sandwiched between the two reference rods, which are heated and cooled, respectively. From a steady-state temperature distribution inside the rods, the thermal conductivity of the sample is obtained by using Fourier's law.

In this method, a one-dimensional heat flow from the heated to the cooled section of the rod is assumed. Therefore, an attention is required when this method is applied for the measurement of an effective thermal conductivity of a composite sample. This is because a difference in thermal conductivity between components of the composite sample may cause a constriction/spreading heat flow inside the rods. The effect of the constriction/spreading heat flow on the measurement results would be conspicuous when the difference in thermal conductivity between the components of the composite sample is very large. Concerning the constriction/spreading heat flow and thermal resistance, a detailed review article was presented by Yovanovich and Marotta [4]. Recently, Rahmani et al. [5] showed the analytical results on the thermal spreading resistance of a curved-edge heat spreader. Moreover, Kumar and Tariq [6] showed the experimental results on the constriction/spreading heat transfer characteristics between curvilinear contacts of rods. Up to now, many studies have been conducted on the constriction/spreading resistances. However, to the authors' knowledge, sufficient information has not been published on the constriction/ spreading heat flow inside the rods caused by the composite sample like a polymer board having thermal vias.

The thermal via is one of the options to enhance heat transfer through a board of low thermal conductivity. As mentioned in the authors' previous paper [7], because of an increase in heat dissipation from electronics components mounted on a printed circuit board (PCB), the thermal via has been used to enhance the heat transfer through the PCB. In many cases, the thermal via is a small hollow pillar made of copper and an array of the thermal vias is embedded in the board. Since the thermal conductivity of the thermal via is about 1000 times higher than that of the board, the thermal via serves as a conductive path of heat flow through the board. In addition to the PCB, the thermal via has been also used for polymer heat pipes. Wits and Kok [8] developed a polymer heat pipe using the PCB, while liquid-crystal polymer films were employed by Oshman et al. [9] as a casing material. Because the thermal conductivities of these polymer materials were much lower than those of metals, the thermal vias were placed at a heated and a cooled section of their polymer heat pipes. Moreover, Yang et al. [10] also used the thermal via for their polymer heat pipe and their experimental results showed that the use of the thermal via reduced a thermal resistance by $20 \%$ $25 \%$.

This paper describes numerical analyses on the heat transfer characteristics of the two rods having the composite board in between. The present analyses dealt with a polymer-based composite board having the thermal vias. As mentioned 
earlier, sufficient information has not been published on the constriction/ spreading heat flow inside the rods caused by such a composite board. Like the authors' previous study [7], the numerical analyses were conducted by changing the number and size of the thermal vias in the board. From the numerical results of temperature distributions, the constriction/spreading resistance of the rods was obtained. A simple equation was also proposed to evaluate the constriction/ spreading resistance. Furthermore, the discussion was also made on an effective thermal conductivity of the composite board evaluated with the two rods.

\section{Analytical Methods}

Figure 1 shows an analytical model. As shown in Figure 1(a), a composite board was placed between an upper and a lower rod, and their heat transfer characteristics were numerically analyzed in a three-dimensional $x, y, z$ coordinate system. The two rods were same in size and had a same thermal conductivity of $\lambda_{r}$ The composite board was a model of a polymer board having thermal vias. As shown in Figure 1(b), the composite board had a thickness of $\delta$ and consisted of two materials having a high, $\lambda_{h}$, and a low, $\lambda_{b}$ thermal conductivity. A uniform heat flux, $q_{h}$, was applied on a top surface of the upper rod while a bottom surface of the lower rod was maintained at a uniform temperature, $T_{\mathcal{c}}$ Outer surfaces of the model were thermally insulated except the heated and the cooled section. The temperature distributions of the model were obtained by solving a heat conduction equation given by

$$
\nabla \cdot\left(\lambda_{j} \nabla T\right)=0 \quad(j=h, l, r)
$$

with boundary conditions of

$$
-\lambda_{r}(\partial T / \partial z)=q_{h} \text { for the heated section }
$$

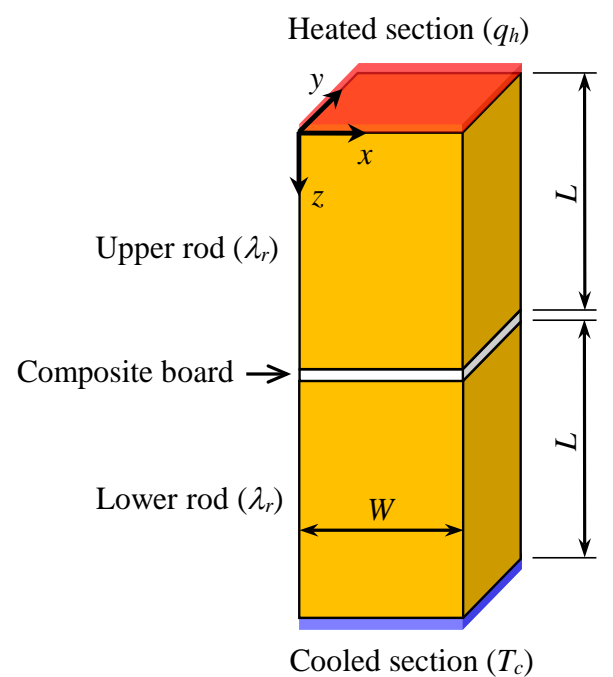

(a)

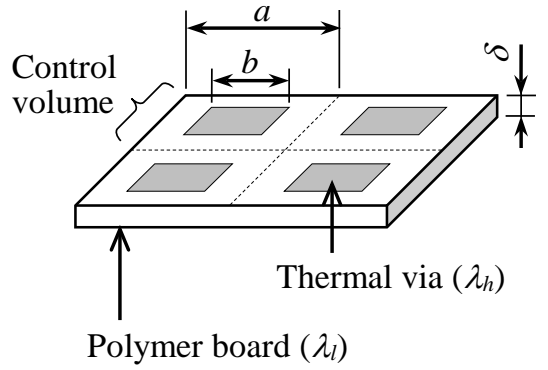

(b)

Figure 1. Analytical model. (a) Computational domain; (b) Details of the composite board (Type 1). 


$$
\begin{gathered}
T=T_{c} \text { for the cooled section } \\
\partial T / \partial n=0 \text { for the adiabatic section }
\end{gathered}
$$

where $n$ was a coordinate normal to a boundary surface. The governing equation was discretised by a control volume method.

As shown in Figure 2, four types of arrays of the thermal vias were considered in the present analyses. While a total volume of the thermal vias was kept, the number of the thermal vias, $N$, was increased by dividing them as shown in the figure. The sizes of the thermal vias on each board were the same. Table 1 shows the relation between $N$ and the size of each thermal via. The cross-sectional areas of the rods and the board were the same $(32 \mathrm{~mm} \times 32 \mathrm{~mm})$. The lengths of the upper and the lower rod were both $45 \mathrm{~mm}$. The numerical results to be presented in the next section were obtained under the heating/cooling conditions of $q_{h}=5.0 \mathrm{~W} / \mathrm{cm}^{2}$ and $T_{c}=20^{\circ} \mathrm{C}$.

From the numerical results of the temperature distribution of the model, the thermal resistance of the board, $R_{b}$, was evaluated by

$$
R_{b}=R_{t}-2 R_{r}
$$

where $R_{t}$ was the total thermal resistance of the model and $R_{r}$ the thermal resistance of each rod. $R_{t}$ and $R_{r}$ were expressed respectively as

$$
\begin{gathered}
R_{t}=\frac{T_{h}-T_{c}}{q_{h} W^{2}} \\
R_{r}=\frac{L}{\lambda_{r} W^{2}}
\end{gathered}
$$

where $T_{h}$ was the temperature at the top surface of the upper rod. $L$ and $W$ were the length $(=45 \mathrm{~mm})$ and the width $(=32 \mathrm{~mm})$ of the rod, respectively.

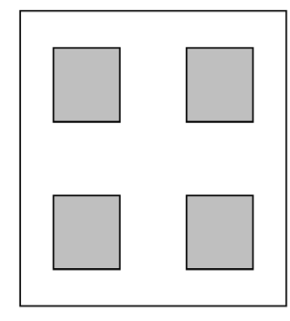

(a)

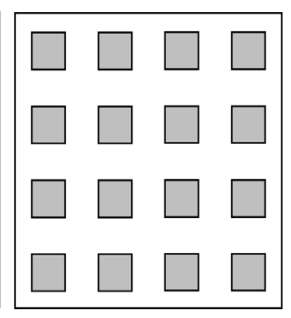

(b)

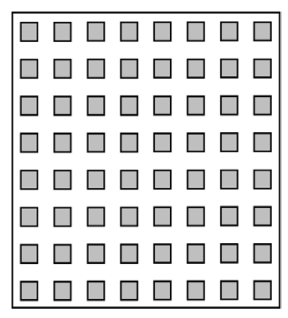

(c)

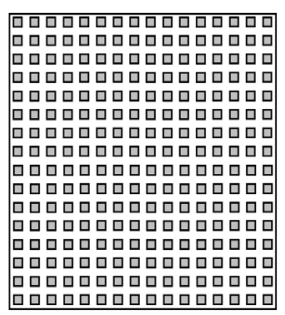

(d)

Figure 2. Arrays of the thermal vias. (a) Type 1; (b) Type 2; (c) Type 3; (d) Type 4.

Table 1. Number and size of the thermal vias.

\begin{tabular}{ccc}
\hline Type & Number & Size \\
\hline 1 & 4 & $8 \mathrm{~mm} \times 8 \mathrm{~mm}$ \\
2 & 16 & $4 \mathrm{~mm} \times 4 \mathrm{~mm}$ \\
3 & 64 & $2 \mathrm{~mm} \times 2 \mathrm{~mm}$ \\
4 & 256 & $1 \mathrm{~mm} \times 1 \mathrm{~mm}$ \\
\hline
\end{tabular}




\section{Results and Discussion}

Figure 3 shows the temperature distributions inside the model. The numerical results were obtained at the thermal conductivities of $\lambda_{h}=400 \mathrm{~W} /(\mathrm{m} \cdot \mathrm{K}), \lambda_{l}=$ $0.40 \mathrm{~W} /(\mathrm{m} \cdot \mathrm{K})$ and $\lambda_{r}=113 \mathrm{~W} /(\mathrm{m} \cdot \mathrm{K})$, which correspond to the thermal condutivities of a copper, a polymer and a brass, respectively. The thickness of the composite board, $\delta$, was $2.0 \mathrm{~mm}$. The temperature distributions at the cross section of $y=x$ for Type $1(N=4)$ to Type $4(N=256)$ are compared in this figure. Since the top surface of the upper rod was heated while the bottom surface cooled, the heat flowed downward from the heated to the cooled section. Onedimensional heat flows were found inside the upper and the lower rods. However, because of a large difference in thermal conductivity between the thermal via, $\lambda_{h}$, and the polymer, $\lambda_{b}$ in the board, the heat flow was constricted in the upper rod and spread in the lower rod near the board. It was also found that the constriction/spreading of the heat flow became smaller with $N$, resulting in a smaller temperature difference between the heated and the cooled section of the rod.

Figure 4 shows the relation between the thermal resistance of the composite

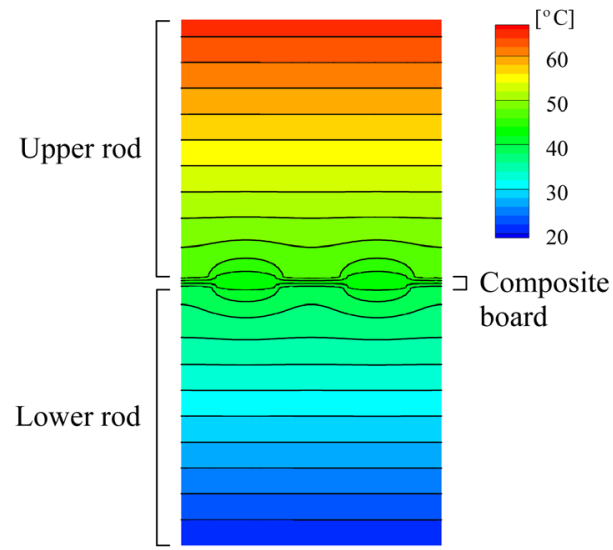

(a)

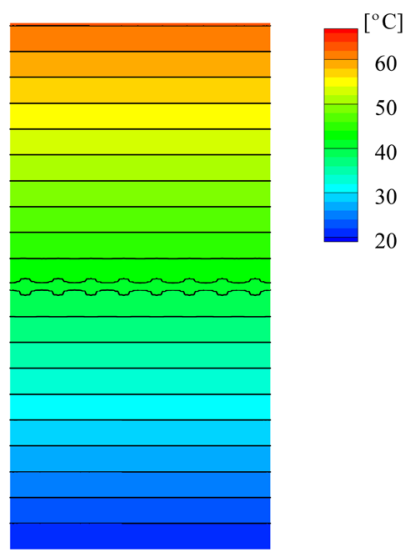

(c)

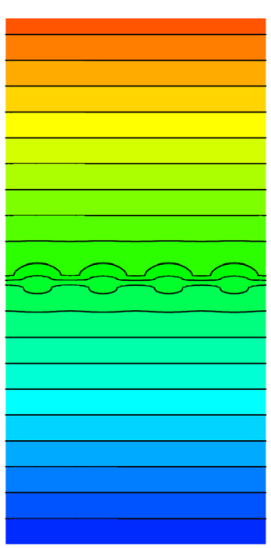

(b)

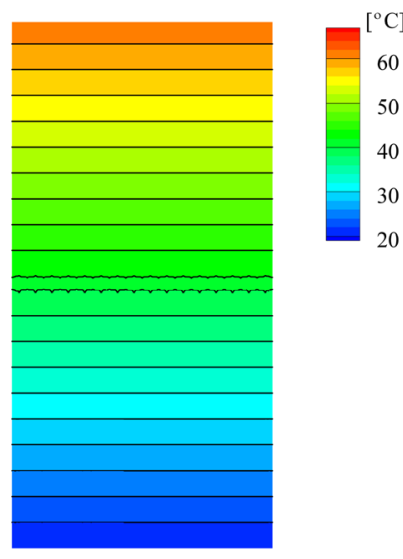

(d)

Figure 3. Temperature distributions at the cross section of $y=x$ inside the model $\left(\lambda_{h}=\right.$ $\left.400 \mathrm{~W} /(\mathrm{m} \cdot \mathrm{K}), \lambda_{I}=0.40 \mathrm{~W} /(\mathrm{m} \cdot \mathrm{K}), \lambda_{r}=113 \mathrm{~W} /(\mathrm{m} \cdot \mathrm{K}), \delta=2.0 \mathrm{~mm}\right)$. (a) Type 1; (b) Type 2; (c) Type 3; (d) Type 4. 


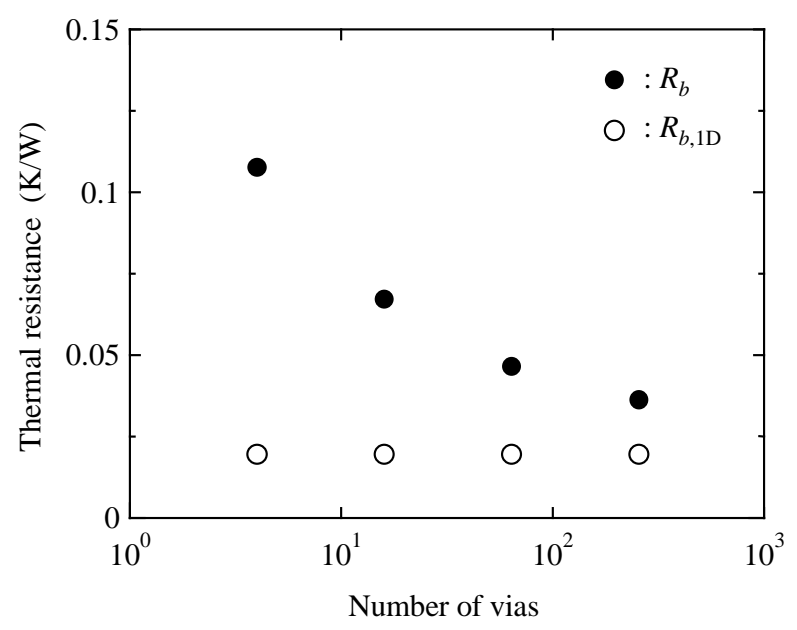

Figure 4. Thermal resistance of the composite board $\left(\lambda_{h}=400\right.$ $\left.\mathrm{W} /(\mathrm{m} \cdot \mathrm{K}), \lambda_{I}=0.40 \mathrm{~W} /(\mathrm{m} \cdot \mathrm{K}), \lambda_{r}=113 \mathrm{~W} /(\mathrm{m} \cdot \mathrm{K}), \delta=2.0 \mathrm{~mm}\right)$.

board, $R_{b}$, and the number of the thermal vias, $N$, at $\lambda_{h}=400 \mathrm{~W} /(\mathrm{m} \cdot \mathrm{K}), \lambda_{l}=0.40$ $\mathrm{W} /(\mathrm{m} \cdot \mathrm{K}), \lambda_{r}=113 \mathrm{~W} /(\mathrm{m} \cdot \mathrm{K})$ and $\delta=2.0 \mathrm{~mm}$. It is noted that $R_{b}$ includes the constriction/spreading resistance of the rods. Moreover, when a one-dimensional heat flow inside the composite board was assumed and an equivalent thermal circuit for parallel two thermal resistances was considered, the thermal resistance of the board, $R_{b, 1 \mathrm{D}}$, was calculated by

$$
R_{b, 1 \mathrm{D}}=\left(\frac{\lambda_{h} A_{h}}{\delta}+\frac{\lambda_{1} A_{1}}{\delta}\right)^{-1}
$$

where $A_{h}$ and $A_{l}$ were the cross-sectional areas of the thermal vias and the polymer in the board, respectively. The thermal resistance obtained by Equation (8) is also shown in Figure 4. Because $R_{b, 1 \mathrm{D}}$ does not consider the constriction/ spreading resistance of the rods, $R_{b, 1 \mathrm{D}}$ was smaller than $R_{b}$ and not affected by $N$. Moreover, it was also found that the difference between $R_{b}$ and $R_{b, 1 \mathrm{D}}$ became smaller with the increase in $N$. The constriction/spreading resistance of the rods, $R_{c s}$, was evaluated by

$$
R_{c s}=R_{b}-R_{b, 1 \mathrm{D}}
$$

The relations between the constriction/spreading resistance, $R_{c s}$ and the number of the thermal vias, $N$, are shown in Figure 5 and Figure 6, where the thermal conductivities of the rods, $\lambda_{t}$, and the thermal vias, $\lambda_{h}$, were changed respectively at $\lambda_{l}=0.40 \mathrm{~W} /(\mathrm{m} \cdot \mathrm{K})$ and $\delta=2.0 \mathrm{~mm}$. From Figure 5 , it was found that the relation between $R_{c s}$ and $N$ was strongly affected by $\lambda_{r}$ confirming that the thermal conductivity of the rod is a factor dominating the constriction/ spreading resistance of the rods. From Figure 6, it was confirmed that the relation between $R_{c s}$ and $N$ was hardly affected by $\lambda_{h}$ in the present calculation range from $\lambda_{h}=100 \mathrm{~W} /(\mathrm{m} \cdot \mathrm{K})$ to $\lambda_{h}=400 \mathrm{~W} /(\mathrm{m} \cdot \mathrm{K})$.

As shown in Figure 1(b) by dashed lines, a square control volume was considered for each thermal via, and then the following simple equation was derived 

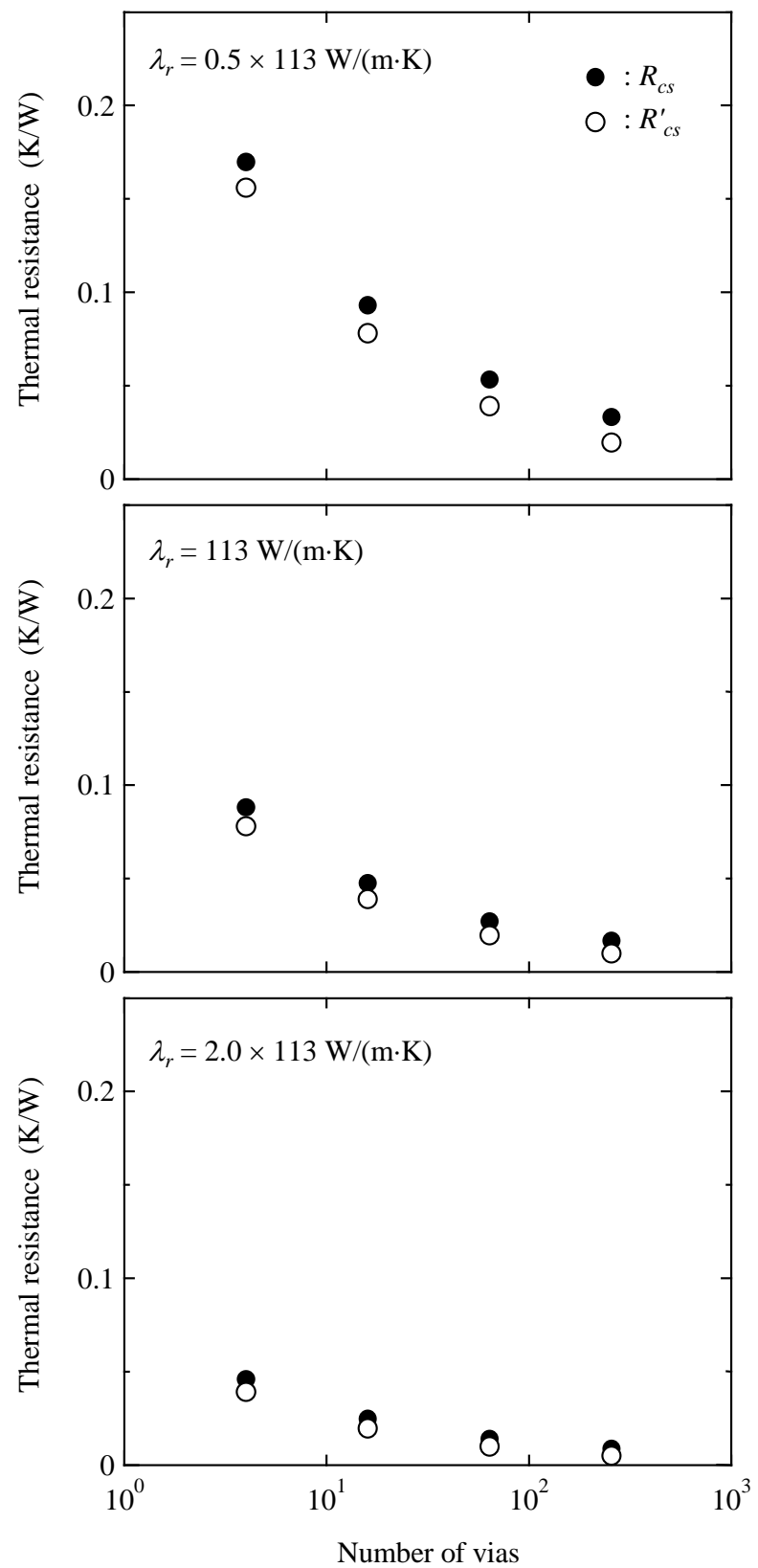

Figure 5. Constriction/spreading resistance of the rods: effect of the thermal conductivity of the rods, $\lambda_{r}\left(\lambda_{h}=400 \mathrm{~W} /(\mathrm{m} \cdot \mathrm{K})\right.$, $\left.\lambda_{l}=0.40 \mathrm{~W} /(\mathrm{m} \cdot \mathrm{K}), \delta=2.0 \mathrm{~mm}\right)$.

to evaluate the constriction/spreading resistance of the rods, $R_{c s}^{\prime}$ :

$$
R_{c s}^{\prime}=\frac{a-b}{N \sqrt{\pi} a b \lambda_{r}}
$$

where $a$ and $b$ were the side lengths of the square control volume and the thermal via, respectively (see Figure 1(b)). The details of the derivation of Equation (10) are described in Appendix. The thermal resistance obtained by Equation (10) is also shown in Figure 5 and Figure 6. In the present calculation range, fairly 


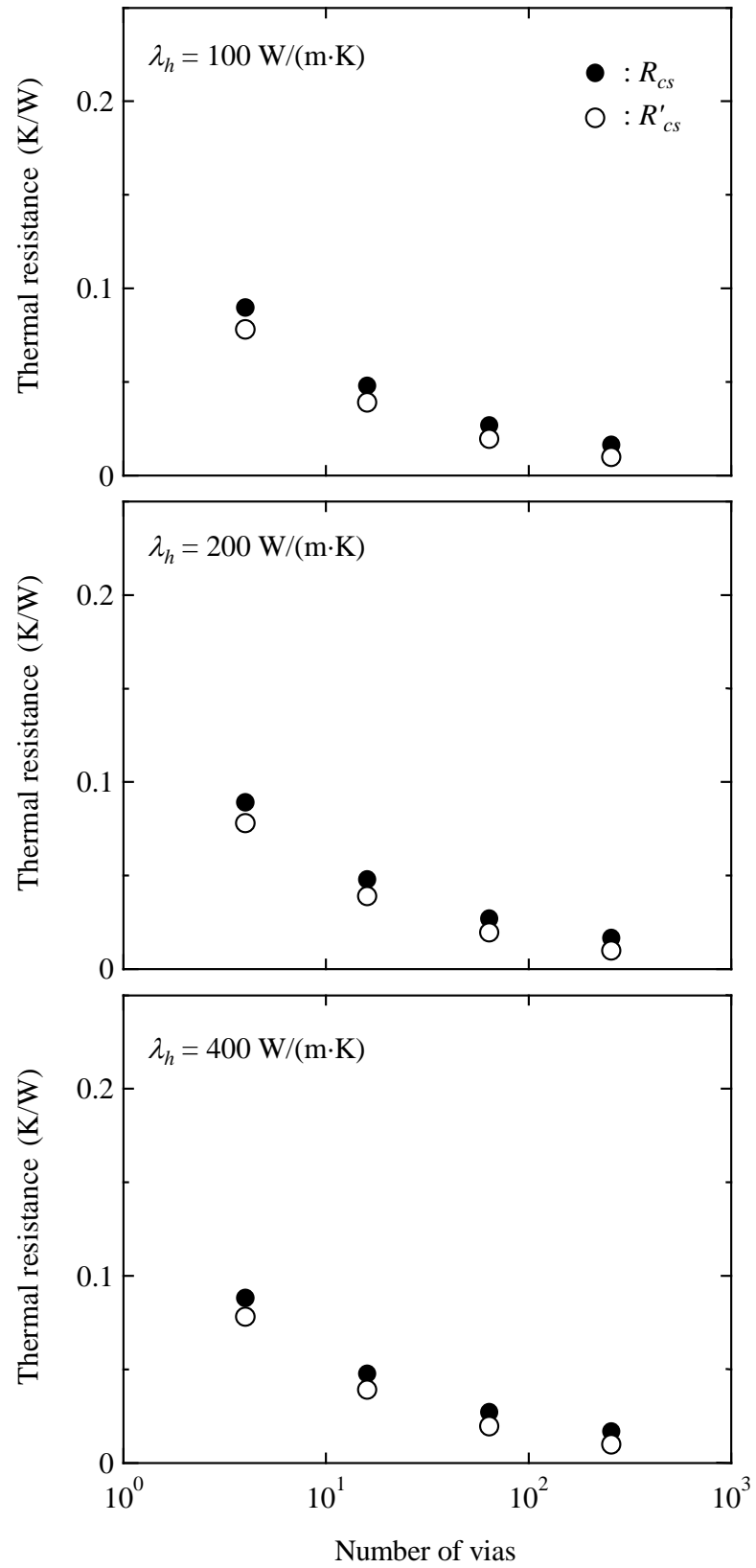

Figure 6. Constriction/spreading resistance of the rods: effect of the thermal conductivity of the thermal vias, $\lambda_{h}\left(\lambda_{l}=0.40\right.$ $\left.\mathrm{W} /(\mathrm{m} \cdot \mathrm{K}), \lambda_{r}=113 \mathrm{~W} /(\mathrm{m} \cdot \mathrm{K}), \delta=2.0 \mathrm{~mm}\right)$.

good agreements were obtained between $R_{c s}$ and $R_{c s}^{\prime}$ confirming the validity of Equation (10) for the evaluation of the constriction/spreading resistance of the rods.

The relation between $R_{c s}$ and $N$ is shown in Figure 7, where the thickness of the composite board, $\delta$, was changed at $\lambda_{h}=400 \mathrm{~W} /(\mathrm{m} \cdot \mathrm{K}), \lambda_{l}=0.40 \mathrm{~W} /(\mathrm{m} \cdot \mathrm{K})$ and $\lambda_{r}=113 \mathrm{~W} /(\mathrm{m} \cdot \mathrm{K})$. Moreover, the ratio of $R_{c s}$ to $R_{b}$ was calculated and the relation between $R_{c s} / R_{b}$ and $N$ is shown in Figure 8. It was found that $R_{c s}$ was hardly affected by $\delta$ while $R_{c s} / R_{b}$ decreased with the increase in $\delta$ implying that 


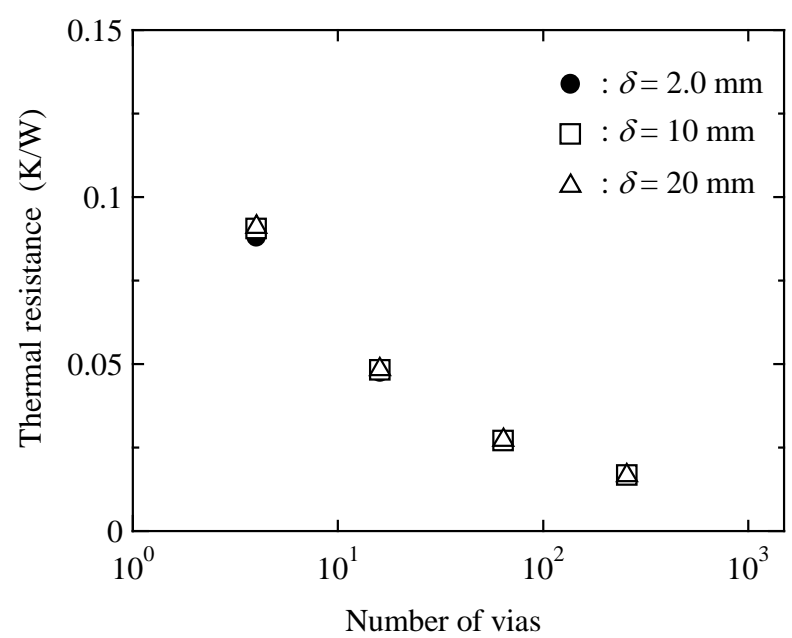

Figure 7. Constriction/spreading resistance of the rods: effect of the thickness of the composite board, $\delta\left(\lambda_{h}=400 \mathrm{~W} /(\mathrm{m} \cdot \mathrm{K})\right.$, $\left.\lambda_{l}=0.40 \mathrm{~W} /(\mathrm{m} \cdot \mathrm{K}), \lambda_{r}=113 \mathrm{~W} /(\mathrm{m} \cdot \mathrm{K})\right)$.

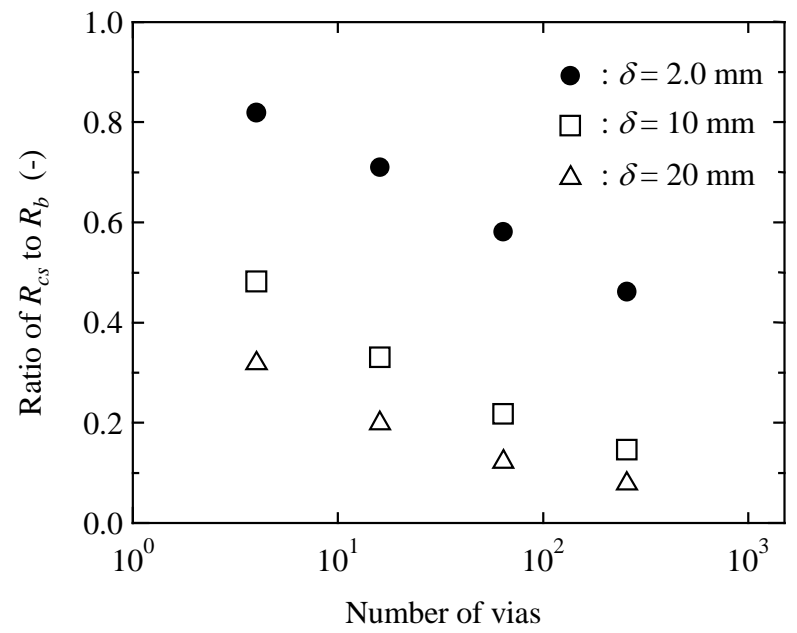

Figure 8. Ratio of the constriction/spreading resistance of the rods to the thermal resistance of the composite board $\left(\lambda_{h}=\right.$ $\left.400 \mathrm{~W} /(\mathrm{m} \cdot \mathrm{K}), \lambda_{l}=0.40 \mathrm{~W} /(\mathrm{m} \cdot \mathrm{K}), \lambda_{r}=113 \mathrm{~W} /(\mathrm{m} \cdot \mathrm{K})\right)$.

the effect of $R_{c s}$ on $R_{b}$ became smaller with the increase in $\delta$. This is because of the increase in $R_{b}$ with $\delta$. The effective thermal conductivity of the composite board, $\lambda_{\text {eff }}$ was calculated by

$$
\lambda_{\text {eff }}=\frac{\delta}{R_{b} W^{2}}
$$

Moreover, when a one-dimensional heat flow inside the composite board was assumed, the effective thermal conductivity of the board, $\lambda_{\text {eff,1D }}$, was calculated by

$$
\lambda_{\text {eff }, 1 \mathrm{D}}=\frac{\delta}{R_{b, 1 \mathrm{D}} W^{2}}
$$

It is noted that $R_{b}$ in Equation (11) was obtained from the numerical results 
while $R_{b, 1 \mathrm{D}}$ in Equation (12) was calculated by Equation (8).

Figure 9 shows the relation between $\lambda_{\text {eff }}$ and $N$ when $\delta$ was changed at $\lambda_{h}=$ $400 \mathrm{~W} /(\mathrm{m} \cdot \mathrm{K}), \lambda_{I}=0.40 \mathrm{~W} /(\mathrm{m} \cdot \mathrm{K})$ and $\lambda_{r}=113 \mathrm{~W} /(\mathrm{m} \cdot \mathrm{K})$. The effective thermal conductivity obtained by Equation (12) is also shown in this figure. In case of the thinner board of $\delta=2.0 \mathrm{~mm}$, a relatively large difference was found between $\lambda_{\text {eff }}$ and $\lambda_{\text {eff }, 1 \mathrm{D}}$ due to the constriction/spreading heat flow inside the rods. However, because the effect of $R_{c s}$ on $R_{b}$ was reduced, it was also found that the difference between $\lambda_{\text {eff }}$ and $\lambda_{\text {eff,iD }}$ became smaller with the increase in $\delta$. In the present calculation range, the difference between $\lambda_{\text {eff }}$ and $\lambda_{\text {eff,iD }}$ was minimum $\left(\lambda_{\text {eff }}=92.4\right.$ $\left.\mathrm{W} /(\mathrm{m} \cdot \mathrm{K}), \lambda_{\text {eff }, 1 \mathrm{D}}=100 \mathrm{~W} /(\mathrm{m} \cdot \mathrm{K})\right)$ when $N=256$ and $\delta=20 \mathrm{~mm}$.

\section{Conclusions}

Numerical analyses were conducted on the heat transfer characteristics of the heated and the cooled rod having the composite board in between. The present analyses dealt with the polymer-based composite board having the thermal vias.

From the numerical results, the constriction/spreading heat flow inside the rods caused by the composite board was clarified. It was found that the constriction/spreading resistance of the rods was strongly affected by the thermal conductivity of the rods as well as the number and size of the thermal vias. A simple equation was also proposed to evaluate the constriction/spreading resistance of the rods. The validity of the simple equation was confirmed by the comparison with the numerical results. The attention is required when the effective thermal conductivity of the composite board is evaluated with the heated and the cooled rod. This is because of the constriction/spreading heat flow inside the rods. It was found that the effect of the constriction/spreading resistance on the effective thermal conductivity became smaller with the increase in the thickness of the

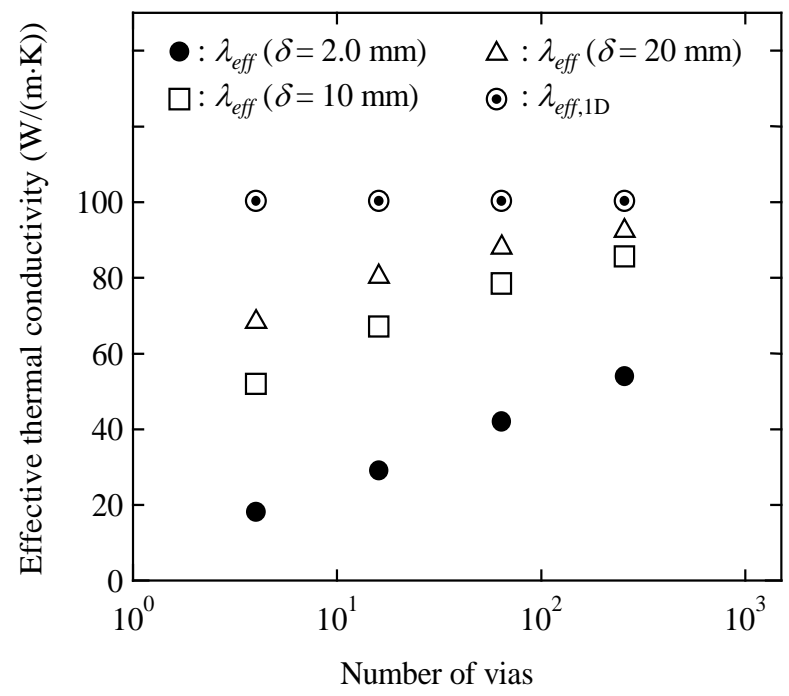

Figure 9. Effective thermal conductivity of the composite board: effect of the thickness of the composite board, $\delta\left(\lambda_{h}=\right.$ $\left.400 \mathrm{~W} /(\mathrm{m} \cdot \mathrm{K}), \lambda_{1}=0.40 \mathrm{~W} /(\mathrm{m} \cdot \mathrm{K}), \lambda_{r}=113 \mathrm{~W} /(\mathrm{m} \cdot \mathrm{K})\right)$. 
composite board as well as the decrease in the size of the thermal vias.

\section{References}

[1] ASTM E1225-04 (2004) Standard Test Method for Thermal Conductivity of Solids by Means of the Guarded-Comparative-Longitudinal Heat Flow Technique. ASTM International, West Conshohocken.

[2] Tomimura, T., Shiotsu, Y., Koito, Y., Ishizuka, M. and Hatakeyama, T. (2011) Evaluation of Effective Thermal Conductivity of Multilayer Printed Circuit Board. Proceedings of 8th ASME/JSME Thermal Engineering Joint Conference, Honolulu, 13-17 March 2011, AJTEC2011-44232.

[3] Sadeghi, E., Hsieh, S. and Bahrami, M. (2011) Thermal Conductivity and Contact Resistance of Metal Foams. Journal of Physics D: Applied Physics, 44, Article ID: 125406. https://doi.org/10.1088/0022-3727/44/12/125406

[4] Yovanovich, M.M. and Marotta, E.E. (2003) Thermal Spreading and Contact Resistances. In: Bejan, A. and Kraus, A.D., Eds., Heat Transfer Handbook, John Wiley \& Sons, Inc., Hoboken, 261-393.

[5] Rahmani, Y., Ganji, D.D. and Bandpy, M.G. (2016) Analytical Study of Thermal Spreading Resistance in Curved-Edge Heat Spreader. Applied Thermal Engineering, 104, 527-533. https://doi.org/10.1016/j.applthermaleng.2016.05.107

[6] Kumar, S. and Tariq, A. (2017) Steady State Experimental Investigation of Thermal Contact Conductance between Curvilinear Contacts Using Liquid Crystal Thermography. International Journal of Thermal Sciences, 118, 53-68. https://doi.org/10.1016/j.ijthermalsci.2017.04.014

[7] Koito, Y., Kubo, Y. and Tomimura, T. (2013) Numerical Analysis of Printed Circuit Board with Thermal Vias: Heat Transfer Characteristics under Nonisothermal Boundary Conditions. Journal of Electronics Cooling and Thermal Control, 3, 136-143. https://doi.org/10.4236/jectc.2013.34015

[8] Wits, W.W. and Kok, J.B.W. (2011) Modeling and Validating the Transient Behavior of Flat Miniature Heat Pipes Manufactured in Multilayer Printed Circuit Board Technology. ASME Journal of Heat Transfer, 133, Article ID: 081401.

[9] Oshman, C., Shi, B., Li, C., Yang, R., Lee, Y.C., Peterson, G.P. and Bright, V.M. (2011) The Development of Polymer-Based Flat Heat Pipes. Journal of Microelectromechanical Systems, 20, 410-417. https://doi.org/10.1109/JMEMS.2011.2107885

[10] Yang, K.S., Yang, T.Y., Tu, C.W., Yeh, C.T. and Lee, M.T. (2015) A Novel Flat Polymer Heat Pipe with Thermal Via for Cooling Electronic Devices. Energy Conversion and Management, 100, 37-44. https://doi.org/10.1016/j.enconman.2015.04.063 


\section{Appendix}

A constriction heat flow in a semi-sphere from the radius $r=r_{1}$ to $r=r_{2}\left(r_{1}>r_{2}\right)$ was considered. The heat flow rate, $Q_{1}$, was expressed as

$$
Q_{1}=2 \pi r_{1} r_{2} \lambda_{r} \frac{\Delta T}{r_{1}-r_{2}}
$$

where $\Delta T$ was the temperature difference between the points at $r=r_{1}$ and $r=r_{2}$. When the number of the constriction heat flow was $N$, the total heat flow rate, $Q_{N}$, was obtained by

$$
Q_{N}=N Q_{1}
$$

From Equations (A1) and (A2), the constriction resistance, $R_{c}^{\prime}$, was obtained as

$$
R_{c}^{\prime}=\frac{\Delta T}{Q_{N}}=\frac{\Delta T}{N Q_{1}}=\frac{r_{1}-r_{2}}{2 N \pi r_{1} r_{2} \lambda_{r}}
$$

The spreading resistance, $R_{s}^{\prime}$, from $r=r_{2}$ to $r=r_{1}$ was also expressed by Equation (A3). Because the cross sections of the control volume and the thermal via were square in shape (see Figure 1(b)), $r_{1}$ and $r_{2}$ were evaluated respectively by

$$
\pi r_{1}^{2}=a^{2}, \pi r_{2}^{2}=b^{2}
$$

Therefore, the constriction/spreading resistance, $R_{c s}^{\prime}$, was obtained as

$$
R_{c s}^{\prime}=R_{c}^{\prime}+R_{s}^{\prime}=\frac{a-b}{N \sqrt{\pi} a b \lambda_{r}}
$$

